\title{
Epithelialization of mouse ovarian tumor cells originating in the fallopian tube stroma
}

\author{
Yuanyuan Hua ${ }^{1,2}$, Pui-Wah Choi ${ }^{2}$, Alexander J. Trachtenberg ${ }^{3}$ Allen C. Ng ${ }^{2}$, \\ Winston P. Kuo ${ }^{3,4}$, Shu-Kay Ng ${ }^{5}$, Daniela M. Dinulescu ${ }^{6}$, Martin M. Matzuk ${ }^{7}$, Ross S. \\ Berkowitz ${ }^{2}$, Shu-Wing $\mathbf{N g}^{2}$ \\ ${ }^{1}$ Department of Obstetrics \& Gynecology, The Second Affiliated Hospital of Chongqing Medical University, Chongqing, \\ PR China \\ ${ }^{2}$ Department of Obstetrics/Gynecology and Reproductive Biology, Brigham and Women's Hospital, Boston, Massachusetts, \\ USA \\ ${ }^{3}$ Harvard Catalyst Laboratory for Innovative Translational Technologies, Harvard Medical School, Boston, Massachusetts, USA \\ ${ }^{4}$ Predicine, Inc., Hayward, California, USA \\ ${ }^{5}$ School of Medicine and Menzies Health Institute Queensland, Griffith University, Nathan, Australia \\ ${ }^{6}$ Department of Pathology, Brigham and Women's Hospital, Boston, Massachusetts, USA \\ ${ }^{7}$ Dan L. Duncan Cancer Center, Baylor College of Medicine, Houston, Texas, USA \\ Correspondence to: Shu-Wing Ng, email: sng@partners.org
}

Keywords: ovarian cancer, fallopian tube

Received: May 11,2016 Accepted: August 13, 2016

Published: September 01, 2016

\section{ABSTRACT}

Epithelial ovarian carcinoma accounts for $90 \%$ of all ovarian cancer and is the most deadly gynecologic malignancy. Recent studies have suggested that fallopian tube fimbriae can be the origin of cells for high-grade serous subtype of epithelial ovarian carcinoma (HGSOC). A mouse HGSOC model with conditional Dicer-Pten double knockout (Dicer-Pten DKO) developed primary tumors, intriguingly, from the fallopian tube stroma. We examined the growth and epithelial phenotypes of the Dicer-Pten DKO mouse tumor cells contributable by each gene knockout. Unlike human ovarian epithelial cancer cells that expressed full-length E-cadherin, the Dicer-Pten DKO stromal tumor cells expressed cleaved E-cadherin fragments and metalloproteinase 2, a mixture of epithelial and mesenchymal markers. Although the Dicer-Pten DKO tumor cells lost the expression of mature microRNAs as expected, they showed high levels of tRNA fragment expression and enhanced AKT activation due to the loss of PTEN function. Introduction of a Dicer1-expressing construct into the DKO mouse tumor cells significantly reduced DNA synthesis and the cell growth rate, with concurrent diminished adhesion and ZO1 epithelial staining. Hence, it is likely that the loss of Dicer promoted mesenchymal-epithelial transition in fallopian tube stromal cells, and in conjunction with Pten loss, further promoted cell proliferation and epithelial-like tumorigenesis.

\section{INTRODUCTION}

Epithelial ovarian cancer is the deadliest of all gynecologic malignancies and is the fifth leading cause of cancer death in females in the United States [1]. Because the lack of a highly specific early detection marker and frequent relapses, the majority of ovarian cancer patients are usually diagnosed at an advanced stage and have a 5 -year survival rate of about 30\% [1]. High-grade serous ovarian carcinoma (HGSOC) is the most common and aggressive type of ovarian neoplasm with epithelial cells resembling those of fallopian tube, comprising about $50 \%$ of primary epithelial ovarian tumors [2]. Historically, ovarian surface epithelial (OSE) cells on the ovarian surface epithelium and the associated cortical inclusion cysts have been suggested as the cells of origin for ovarian cancer $[3,4]$. Recent studies, however, have suggested that epithelial cells in the distal fallopian tube can be the cells of origin for the development of HGSOC. Serous tubal intraepithelial carcinomas (STIC) with both $p 53$ mutations 
and expression of $\gamma$-H2AX, evidence of DNA damage that is frequently observed in HGSOC, are proposed as a potential precursor for HGSOC. [5-8].

Several mouse models with genomic manipulations in specific organ sites have been established for ovarian tumors originating from ovarian surface epithelia [912] and fallopian tube [13], respectively. Mechanistic studies of these mouse models may provide insights into the mechanisms by which native human ovarian cancer develops and is regulated. One recent mouse model employed anti-Muüllerian hormone receptor type 2-directed Cre (Amhr2-Cre) to specifically knockout both Dicer and Pten genes in the mouse female reproductive tract [14]. The Dicer-Pten DKO (Dicerfoxffox Pten flox flox $A m h r 2^{\text {cre/ }+}$ ) mice developed early serous carcinomas in the fallopian tube, which mimicked the morphology and clinical manifestation in human HGSOC. However, intriguingly, the primary epithelial tumors were found to arise from cells of a mesenchymal lineage in the stroma of the fallopian tube [14]. The contribution of Pten dysregulation in ovarian cancer has been well researched in human ovarian cancer and mouse models $[9,10,15-17]$, and the tumors arose from epithelial cells in the mouse models. But for Dicerl, germline mutations have been found in familial pleuropulmonary blastoma, a rare pediatric lung cancer [18], embryonal rhabdomyosarcomas [19], and familial multinodular goiter [20]. Interestingly, somatic Dicerl hotspot missense mutations with defective Dicerl function in $5 \mathrm{p}$ miRNA production were commonly found in nonepithelial ovarian tumors, in particular in $60 \%$ of Sertoli-Leydig cell tumors, and rarely in epithelial ovarian and endometrial carcinomas [21, 22]. Given the predominance of Dicer 1 mutations in nonepithelial ovarian tumors, the appearance of epithelial HGSOC tumors arising from the fallopian tube stroma in the Dicer-Pten DKO mouse model might be likely due to the loss of Dicer1 function.

Molecular characterization of ovarian tumors and cancer cell lines has shown that they are more epithelial-like than normal ovarian surface epithelia and the derived cell lines [3, 4, 23, 24], which possess both mesenchymal and epithelial characteristics for postovulatory wound healing and tissue homeostasis [3, 25]. The expression of adherens junction protein E-cadherin was elevated in ovarian tumors [26] and ectopic expression of E-cadherin in OSE caused mesenchymalepithelial transition and the resulting cells formed tumors in immunodeficient mice [27, 28]. Our previous sequential in vitro three-dimensional culture models have also shown that E-cadherin function is important for ovarian inclusion cyst formation and ovarian tumor invasion [29]. In this study, we examined the epithelial phenotypes of the Dicer-Pten DKO mouse tumor cells and contribution of each knockout genes in tumor phenotypes.

\section{RESULTS}

\section{Epithelial phenotypes of the Dicer-Pten DKO mouse tumors and cancer cell lines}

We first investigated the epithelial phenotypes of the Dicer-Pten DKO mouse tumors by performing immunohistochemistry for the expression of epithelial and mesenchymal markers (Figure 1A). Both the primary and metastatic tumors stained positive for PAX8, a marker for embryonic Müllerian ducts, human fallopian tubes, and serous subtype of ovarian carcinomas [30]. The tumors also had high expression of cytokeratins. However, the tumors showed modest positive staining of adherens junction protein, E-cadherin, and matrix metalloproteinase-2 (MMP2) that are associated with epithelial-mesenchymal-transition (EMT). We also examined the epithelial phenotypes of the Dicer-Pten DKO fallopian tube tumor-derived cancer cell lines (FTdT172 and FTdT967) together with two mouse cancer cell lines originated from the ovarian surface epithelium, OVdT4306 and OVdT4088, which were derived from $\mathrm{K}-\mathrm{ras}^{\mathrm{G}}{ }^{\mathrm{12D} /+} \mathrm{Pten}^{-/ /}$and $\mathrm{K}-\mathrm{ras}^{\mathrm{G} 12 \mathrm{D} /+} \mathrm{TP} 53^{-/-}$mice, respectively, [9]. We also employed two human ovarian cancer cell lines, OVCA432 and MCAS, in the comparison. Western blot analysis of the cell lysates (Figure 1B) showed that all cancer cell lines except OVCA432 expressed epithelial cytokeratins. The human ovarian cancer cell lines expressed high levels of full-length E-cadherin proteins, whereas the mouse ovarian tumor- and fallopian tube tumor-derived cancer cell lines expressed only E-cadherin protein fragments with smaller molecular weights. Furthermore, as reported before [29], the human ovarian cancer cells did not express MMP2, which was expressed in the mouse tumor cell lines. Within the mouse tumor cell lines, the ovarian tumor-derived OVdT4088 and OVdT4306 cancer cells expressed the TGF $\beta$ downstream target phosphorylated Smad2, while the Dicer-Pten DKO cancer cell lines showed very little expression. Instead, the Dicer-Pten DKO cancer cell lines had higher expression of TGF $\beta$ downstream transcription factors Slug and Snail. Hence, the expression analysis showed that the Dicer-Pten DKO mouse fallopian tube tumors and cancer cells expressed a mixture of epithelial and mesenchymal markers, that were very distinct from human epithelial ovarian cancer cells.

\section{Investigation of cell growth and small RNA expression phenotypes of the Dicer-Pten DKO mouse tumors and cancer cell lines}

As HGSOC is a highly aggressive tumor, we compared the growth rate among the mouse tumor cell lines (Figure 2A). Both Dicer-Pten DKO cancer cell lines and the OVdT4306 cancer line showed enhanced growth rate compared with the $\mathrm{K}-\mathrm{ras}^{\mathrm{Gl2D} /+} \mathrm{TP} 53^{-/-} \mathrm{OVdT} 4088$ mouse 
A.

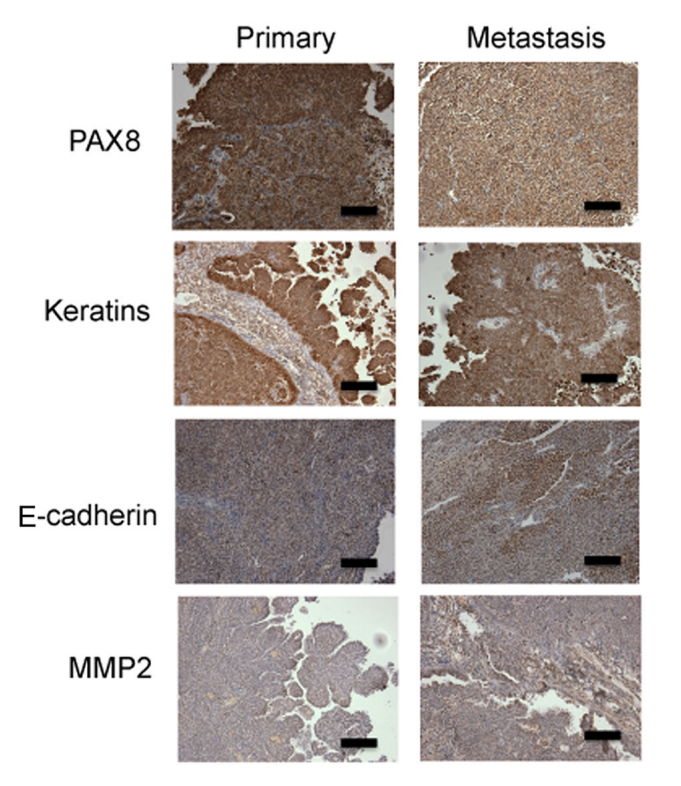

B.

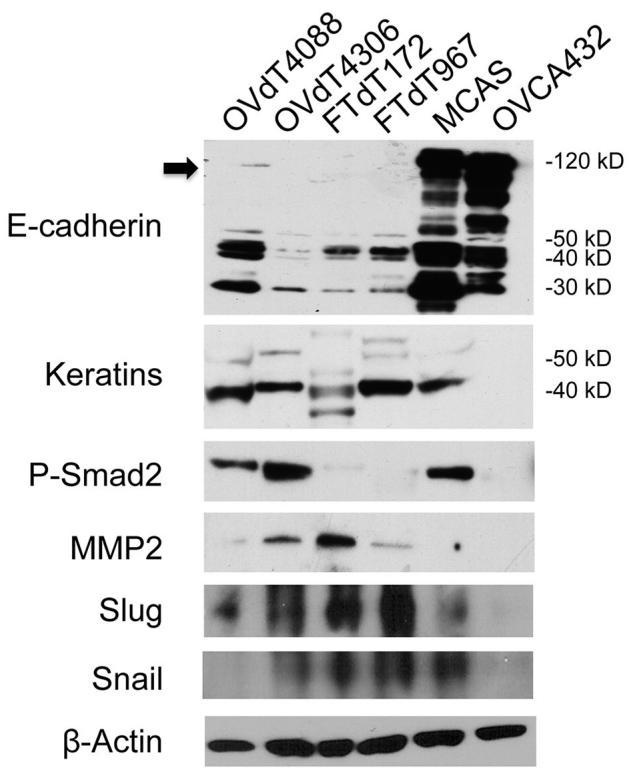

Figure 1: The Dicer-Pten DKO mouse tumor cells express a mixture of epithelial and mesenchymal markers.

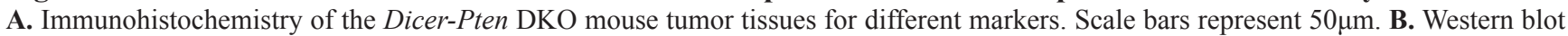
analysis of marker expression in different cell lysates. The position of the full-length E-cadherin is marked by an arrowhead. $\beta$-actin was used as loading control.

A.

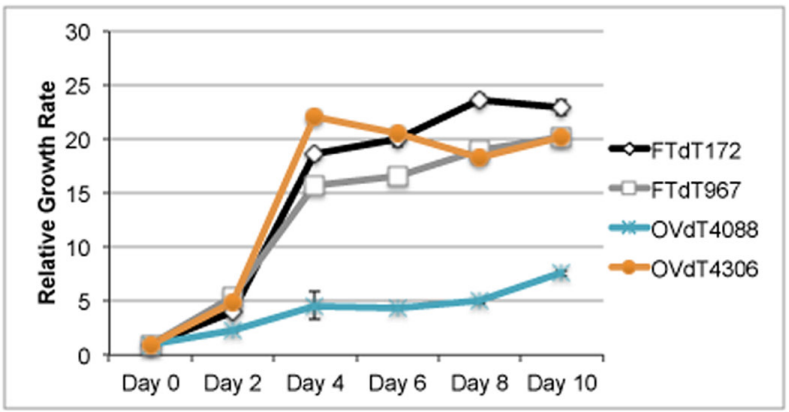

C.

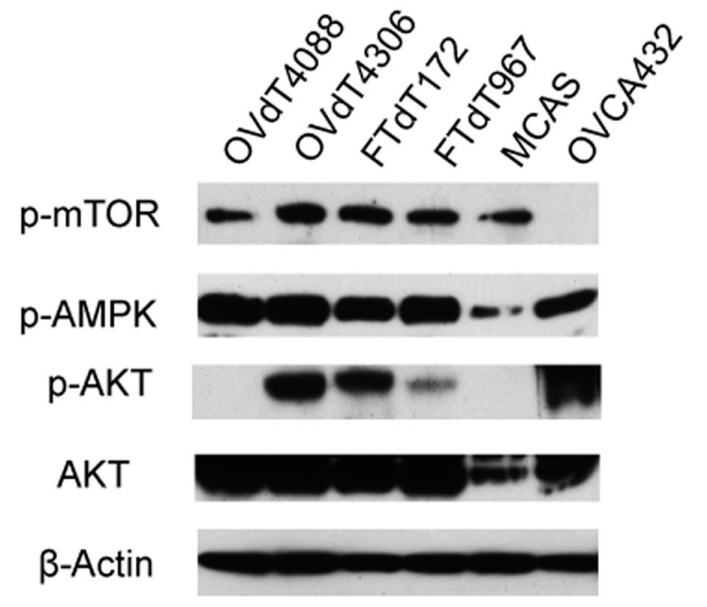

B.

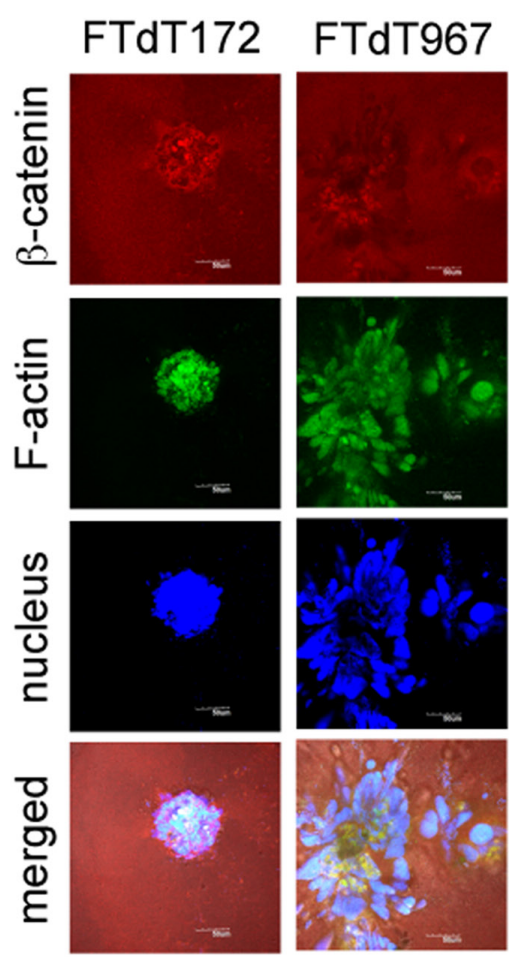

Figure 2: The Pten disruption contributed to the rapid proliferation of the Dicer-Pten DKO mouse cancer cells. A. Cell growth assay to compare the growth rates of different mouse cancer cell lines. B. Confocal microscopic images of three-dimensional in vitro spheres formed by the Dicer-Pten DKO mouse cancer cell lines in Collagen I matrix and stained for $\beta$-catenin (red), F-actin (green) and nucleus (blue). C. Western blot analysis for the expression for growth and metabolic markers. $\beta$-actin was used as loading control. 
cancer cell line. We also tested the two Dicer-Pten DKO cancer cell lines in a sequential three-dimensional culture system which we have previously developed [29]. The FTdT967 line showed more aggressive growth and invaded into the collagen I extracellular matrix after 3 days of growth (Figure 2B), suggesting that this line is derived from a tumor that may have a more aggressive phenotype. Both Dicer-Pten DKO tumor lines and the OVdT4306 cancer cell line shared genomic Pten deletion, which affected the $\mathrm{PI} 3 \mathrm{~K} / \mathrm{AKT}$ key metabolic and cell growth pathway. We investigated the status of AKT pathway in different cancer cell lines by Western blot analysis (Figure 2C). These three mouse cancer cell lines showed increased phospho-AKT expression when compared with the OVdT4088 line, and expectedly, showed stronger expression of the downstream phospho-mTOR target. However, within these four mouse cell lines, there was no change in phospho-AMPK, another master regulator of cell growth and metabolism that can affect mTOR activity [31].
As our preliminary qRT-PCR test showed some minor differences in the miRNA expression in the DicerPten DKO cancer cells (data not shown), we carried out a miRNA transcriptomic profiling of the Dicer-Pten DKO tumor lines 172,177 , and 967 . For comparison, we also determined the miRNA profiles from RNA isolated from normal mouse fallopian tubes, and the two ovary-derived lines. The results of the profiling (Figure 3A and Figure 3B) showed that while most of all the miRNA species examined showed downregulation in the Dicer-Pten DKO tumor lines, there were significantly elevated levels of miR-720 and miR-1937a, b, and c in the DKO tumor lines, which according to miRBase database (http://www. mirbase.org/index.shtml), belonged to tRNA fragments. qRT-PCR performed on the RNA preparations validated the elevated expression of miR-720 tRNA fragments in the three Dicer-Pten DKO tumor lines relative to normal fallopian tube and the other cancer cell lines (Figure 3C, $P<0.05)$. Hence, excessive tRNAs were expressed in

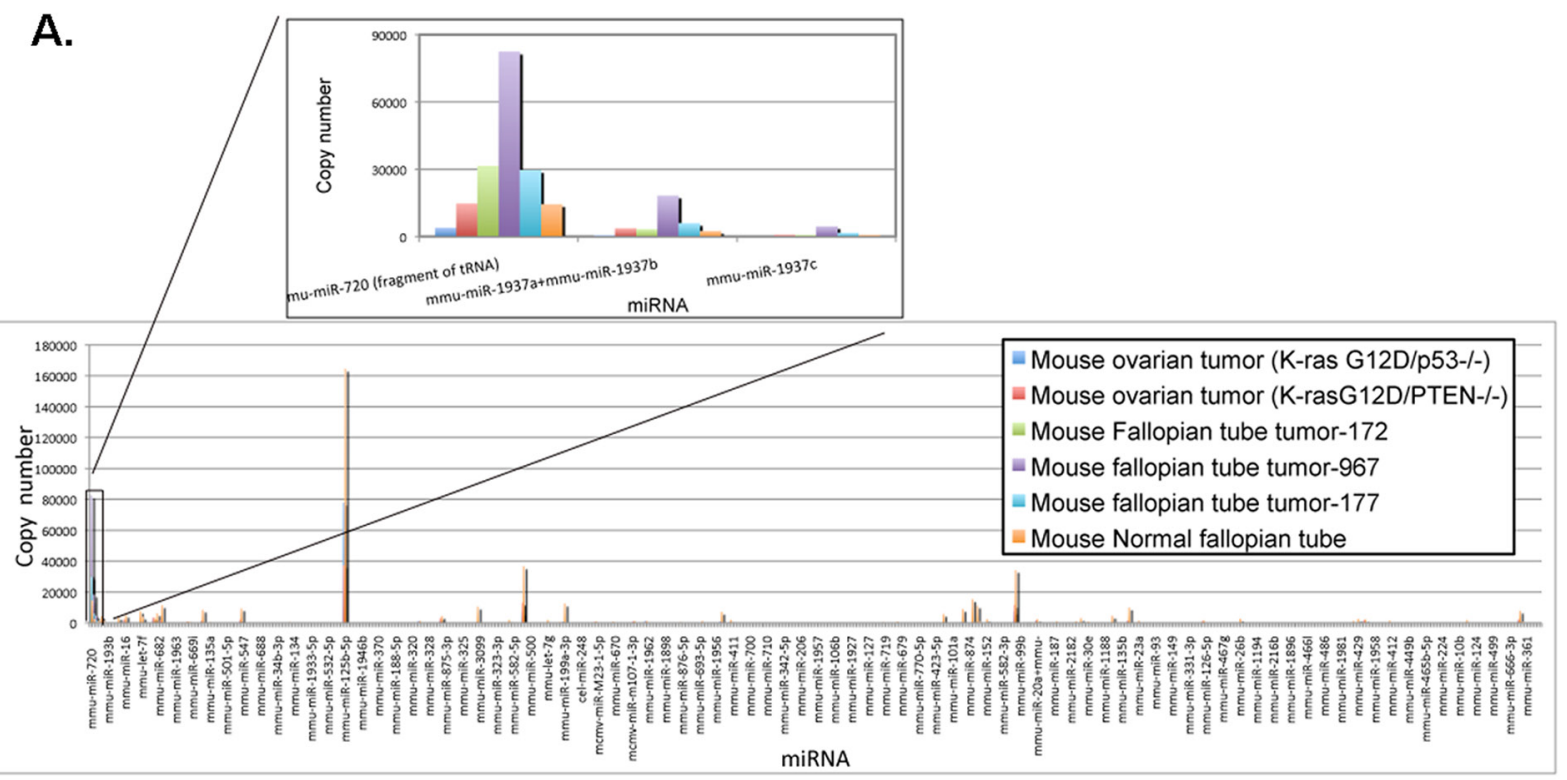

B.

\begin{tabular}{|l|c|c|c|c|c|c|l|}
\hline miRNA & OVdT4088 & OVdT4306 & FTdT172 & FTdT967 & FTdT177 & Normal FT & Remarks \\
\hline mmu-miR-720 (fragment of tRNA) & 3984 & 14694 & 31472 & 82409 & 29633 & 14444 & fragments of tRNAs \\
mmu-miR-1937a+mmu-miR-1937b & 633 & 3649 & 3372 & 18273 & 6018 & 2455 & fragments of tRNAs \\
mmu-miR-1937c & 122 & 831 & 785 & 4617 & 1657 & 690 & fragments of tRNAs \\
mmu-miR-125b-5p & 77672 & 37252 & 13 & 14 & 21 & 164440 & \\
mmu-let-7c & 7410 & 11505 & 6 & 13 & 14 & 34219 & \\
mmu-let-7b & 6804 & 12951 & 11 & 6 & 12 & 36519 & \\
mmu-miR-15b & 2799 & 3590 & 25 & 4 & 6 & 3031 & \\
mmu-let-7e & 1859 & 2267 & 1 & 10 & 6 & 7829 & \\
mmu-miR-20a+mmu-miR-20b & 1723 & 1597 & 6 & 9 & 9 & 2429 & \\
mmu-miR-16 & 1580 & 2359 & 43 & 52 & 29 & 5183 & \\
\hline
\end{tabular}

C.

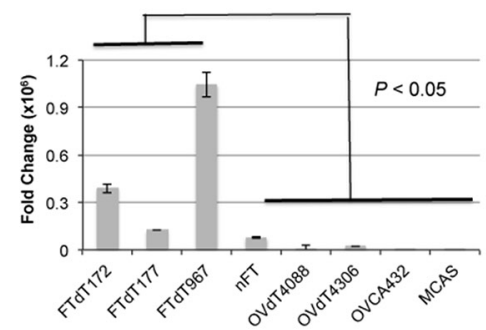

Figure 3: The Dicer-Pten DKO mouse cancer cells expressed elevated levels of tRNA fragments. A. The result of the Nanostring ${ }^{\circledR}$ nCounter miRNA expression assay. The relative expression of mmu-miR-720, mmu-miR-1937a, 1937b, and $1937 \mathrm{c}$ in different cell lines is shown in the inset. B. Normalized counts of the miRNAs representing tRNA fragments that are elevated (red), as well as some other miRNAs that are significantly down-regulated (dark) in the Dicer-Pten DKO mouse cancer cells relative to normal fallopian tube (FT) and ovary-derived tumor cell lines. C. qRT-PCR of mmu-miR-720 in different RNA preparations. 
these DKO lines despite the downregulation of miRNA expression.

\section{Introduction of a Dicer1-expression construct into the Dicer-Pten DKO mouse cancer cells reversed the growth and epithelial phenotypes}

To investigate whether Dicer 1 knockout caused the epithelialization of the mouse fallopian tube stromal cells and affected the growth of the Dicer-Pten DKO cancer cells, a FLAG-tagged Dicerl-expression construct was introduced into both FTdT172 and FTdT967 cells by lentiviral infection and the resulting cells were tested for FLAG-Dicer1 expression (Figure 4A) and cell growth and epithelial phenotypes. Compared to control cells, Dicer 1lentivirus infected cells showed significant reduction in growth rate (Figure 4B). Cell cycle analysis of BrdUand propidium iodide-labeled cells by flow cytometry also showed that the Dicerl-lentivirus infected cells had significant reduction in $\mathrm{S}$ and $\mathrm{G} 2 \mathrm{M}$ phases in the cell cycle (Figure 4C). Intriguingly, Dicer 1 -lentivirus infected FTdT967 cells showed an additional subpopulation of cells between G1 and G2/M. An adhesion assay showed that the Dicer1-lentivirus infected FTdT172 cells showed significant reduced adherence to collagen I extracellular matrix than the control cells (Figure 4D, $P=0.007$ ). The Dicer1-lentivirus infected FTdT967 cells also showed reduced, albeit not significant, cell adherence when compared with the control cells. Nevertheless, they demonstrated loss of membrane-bound expression of the tight junction-associated signaling protein $\mathrm{ZO}-1$ when compared with control cells (Figure 4E).

\section{DISCUSSION}

Recent studies have suggested that the epithelial cells in distal fallopian tube can be the cells of origin for the development of HGSOC [5-8]. A genetically engineered mouse model that disrupted Brcal/Brca2, Tp53, and Pten genes specifically in fallopian tube epithelial cells developed carcinoma that resembled HGSOC [13]. The fact that the tumor development was preventable with the removal of fallopian tube, but not with the removal of the ovaries supported the notion of fallopian tube origin of HGSOC. It is interesting to note that the Dicer-Pten DKO mouse model developed HGSOC-like tumors originating from the stroma of fallopian tube[14], as human HGSOC tumor cells are believed to be derived from epithelial cells that express many epithelial markers [23, 24]. We examined the tumor tissues and derived cancer cell lines and showed that the tumor cells expressed a mixture of epithelial and mesenchymal markers (Figure 1). Actually, the marker expression pattern of the Dicer-Pten DKO tumor cells was similar to that represented by the mouse model established from the mesothelial-like ovarian surface epithelial cells
[9]. While both fallopian tube tumor- and ovarian tumorderived mouse model cell lines expressed epithelial cytokeratins, they expressed only E-cadherin cleaved fragments and the EMT marker MMP2. The mouse cancer cell lines also expressed higher levels of TGF $\beta$ pathway downstream effector phosphorylated Smad2 or transcription factors Slug and Snail. Therefore, while these tumors established epithelial-like properties, they maintained certain mesenchymal characteristics that were distinct from human HGSOC cells. It is also true that the fast growing mouse tumor cells would detach as skin when confluent (data not shown), suggesting some mesenchymal nature of these mouse cancer cells was preserved.

Given the predominance of Dicerl mutations in nonepithelial ovarian tumors [21], it was expected that the knockout of the Dicer1 gene was responsible for the epithelialization of the mouse mesenchymal cells to form epithelial tumors in the Dicer-Pten DKO mouse model. Indeed, introduction of a Dicer l-expressing construct to the Dicer-Pten DKO mouse cancer cells reversed some epithelial phenotypes such as cell adhesion (Figure 4D). Even though the more aggressive growing FTdT967 cells did not show significant reduction in cell adhesion after the reintroduction of Dicer1, the resulting cells nevertheless showed loss of ZO-1 membrane localization of the control cells (Figure 4E). ZO-1 is a tight junctionassociated signaling protein that is important for epithelial cell differentiation [32], the cytoplasmic distribution of ZO-1 in the Dicer 1-reintroduced FTdT967 cells suggested that these cells lost the epithelial phenotype as did the Dicer 1-reintroduced FTdT172 cells. Hence, loss of Dicer 1 in the Dicer-Pten DKO mouse model likely contributed to the epithelialization of stromal cells. Specific knockouts of Dicer1 in other mouse cell types have been reported to affect various cell differentiation and organ development. Disrupting Dicerl function specifically in thyrocytes has shown downregulation of thyrocyte cell adhesion proteins and cell differentiation, which led to severe hypothyroidism and shortened life span [33]. It is also of interest to note that loss of Dicer in epicardiumderived progenitor cells impaired EMT of the progenitor cells and a reduction in epicardial cell proliferation and differentiation into different cell lineages [34]. Hence, it is important to delineate the consequences of Dicer1 dysregulations in a cell context-dependent manner.

We next investigated the contribution of Dicer and Pten gene knockouts on cell growth. Since Pten is known for suppressing PI3K/AKT pathway for cell growth and cell metabolism, the effects of Pten knockout on the activation of AKT and fast cell growth are evident in our assays (Figure 2). Furthermore, our miRNA profiling experiment resulted in the identification of tRNA overexpression in the Dicer-Pten DKO mouse tumors relative to normal fallopian tube cells (Figure 3). PTEN has been reported to act through PI3K/ AKT/mTOR pathway to suppress RNA polymerase 
III-mediated tRNA transcription [35, 36] and enhanced RNA polymerase III activity has been shown to promote oncogenic transformation [37, 38]. Individual tRNA fragments such as miR-720 have also been reported to promote cell migration [39] and represent a signature of breast cancer-derived extracellular vesicles [40]. Hence, it is likely that the loss of Pten in the Dicer-Pten DKO mouse model resulted in upregulation of RNA polymerase
A.

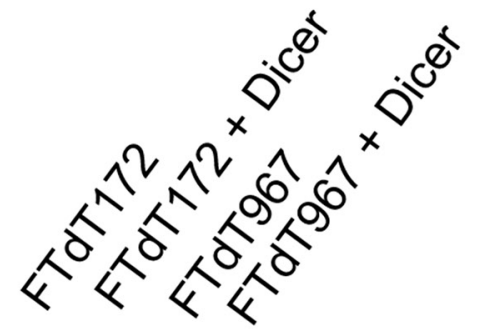

\section{FLAG-Dicer} $\beta$-Actin

B.

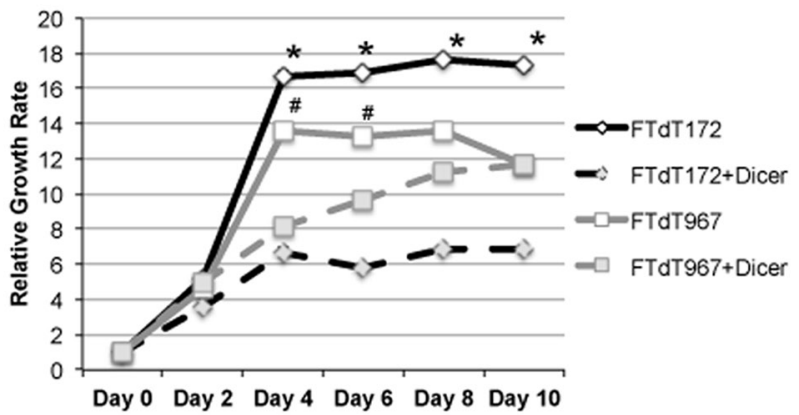

D.

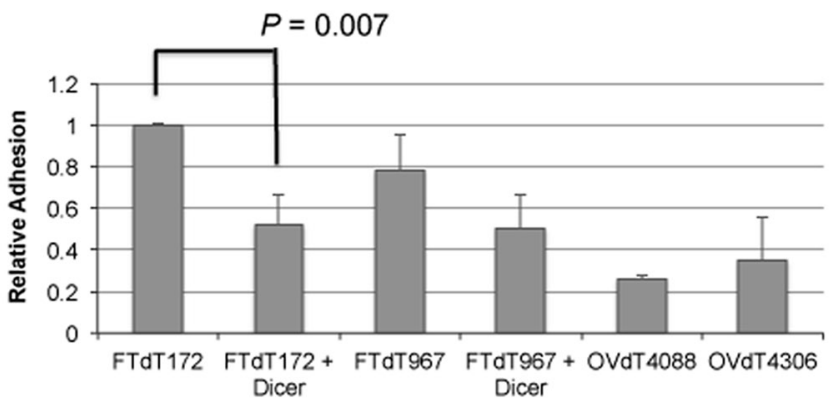

C.
FTdT172

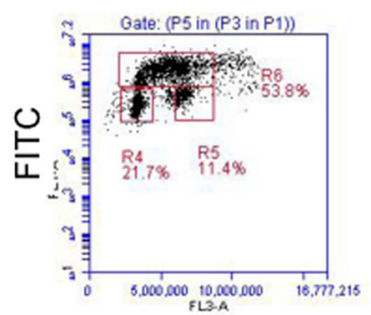

$\mathrm{Pl}$

FTdT967

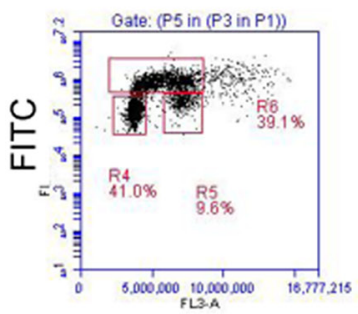

$\mathrm{Pl}$
FTdT172 + dicer

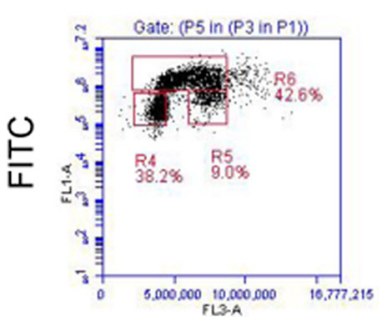

PI

FTdT967 + dicer

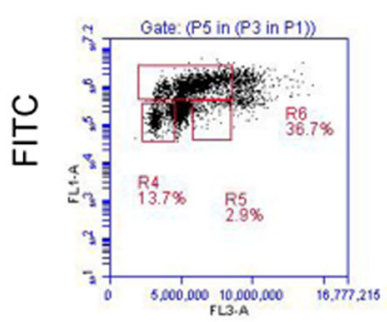

$\mathrm{Pl}$
FTdT967

E.

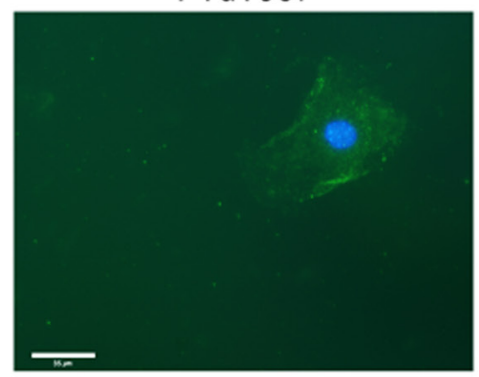

FTdT967 + Dicer

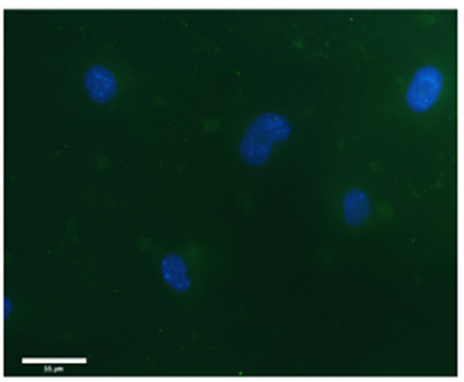

Figure 4: Introduction of a Dicer1-expression construct reversed the epithelial and growth phenotypes of the DicerPten DKO mouse cancer cells. A. Western blot to confirm the expression of FLAG-tagged Dicer1 in the lentivirus-infected cancer cells. $\beta$-actin was used as loading control. B. Cell growth assay to compare the growth rates of control and lentivirus-infected cancer cell lines. ${ }^{*}, P<0.005$; ${ }^{\#}, P<0.02$. C. Flow cytometric density graphs to show the BrdU incorporation and cell cycles of the mouse cancer cell lines. The Y-axis represents the FITC fluorescence of BrdU labeled cells, whereas the X-axis represents the propidium iodide (PI) fluorescence. The boxed areas show the percentages of cell populations in G0/G1 phase (R4), G2/M phase (R5), and S phase (R6), respectively. D. Relative adhesion of different cell lines to collagen I extracellular matrix. The reading of FTdT172 cells was defined as 1. E. Fluorescence micrographs of ZO-1 staining (green) in both FTdT967 cells (top) and FTdT967 cells infected with Dicer1-expressing lentivirus (bottom). The DAPI-stained nuclei were shown in blue. 
III transcription and the tRNA overexpression contributed to oncogenic transformation of the fallopian tube stromal cells.

Besides the contribution by Pten disruption, our studies showed that Dicerl disruption also contributed to the rapid cell proliferation of the DKO mouse cancer cells (Figure 4B and 4C). Our finding is consistent with the report by Chen et al. [22] that cancer cells harboring Dicer1 hotspot mutants that had lost $5 \mathrm{p}$ miRNA biogenesis, showed increased proliferation rate and upregulated expression of cell cycle promoting genes compared to cancer cells with wild-type Dicer1, suggesting the tumor-suppressing effect of Dicer 1 in wildtype cells. Similar to the Dicer 1 hotspot mutant cells, the Dicer-Pten DKO mouse tumor cells showed significant reduced expression of miRNAs such as let-7 and miR125 (Figure 3B), which have been shown to harbor tumor suppressor functions [22, 41].

In conclusion, our studies have delineated the contribution of the Dicer and Pten disruptions to the DKO tumor cells originated in fallopian tube stroma. The loss of Pten contributed to the activated AKT pathway and increased RNA polymerase III transcription, which led to rapid proliferation of the cancer cells. However, disruption of Dicer 1 played a dominant role in epithelialization of fallopian tube stromal cells, and in conjunction with Pten loss, drove these mesenchymal cells to rapid growth and epithelial-like tumorigenesis. Dicer1 dysregulations in epithelial cancers like ovarian and endometrial carcinomas are not as common as in nonepithelial cancers. The results of Cancer Genome Atlas (TCGA) genomic analyses of HGSOC revealed that $2.5 \%$ of HGSOC had alterations of Dicerl gene [42]. Similar to the study of Dicer 1 hotspot mutations in endometrial cancer [43], further studies of the potential role of Dicer 1 dysregulations in affecting stromal cells in fallopian tube will provide insights into this potential route of ovarian cancer development.

\section{MATERIALS AND METHODS}

\section{Cell lines and mouse tumor tissues}

The Dicer-Pten DKO (Dicerflox/flox Pten floxfflox Amhr ${ }^{\text {cre/++}}$ ) mouse tumors and cancer cell lines have been previously described [14]. As a reference, the two mouse ovarian cancer cell lines derived from $K-$ ras $^{\mathrm{G} 12 \mathrm{D} /+} \mathrm{Pten}^{-/}$and $\mathrm{K}-\mathrm{ras}^{\mathrm{G} 12 \mathrm{D} /+}$ $\mathrm{TP}^{2} 3^{-/}$mice, respectively, [9] were used. Ovarian cancer cell lines OVCA432 and MCAS have been described before [44]. The human ovarian cancer cell lines were grown in medium 199 (Sigma-Aldrich, St. Louis, MO) and MCDB 105 (Sigma-Aldrich, St. Louis, MO) (1:1) supplemented with $10 \%$ fetal calf serum (FCS). The mouse tumor cell lines were cultured with DMEM-F12 HAM medium (Life Technologies, Grand Island, NY) plus 10\% FCS.

\section{Immunohistochemistry}

Immunohistochemistry (IHC) was performed on archived formalin-fixed, paraffin-embedded DicerPten DKO mouse tumors tissues. Standard xylene deparaffinization, rehydration with a descending series of ethanol solutions, antigen retrieval (Vector Laboratories, Burlingame, CA), and blocking of endogenous peroxidases in $0.3 \% \mathrm{H}_{2} \mathrm{O}_{2}$ were performed as described before [44]. 3, 3-diaminobenzidine (DAB) horseradish peroxidase substrate kit was used for color development (Vector Laboratories, Burlingame, CA).

\section{Cell growth assay, three-dimensional cultures, and Western blot analysis}

Cell growth study was performed by seeding $5 \times 10^{3}$ cells per well in 96-well microtiter plates and the growth was determined by measuring methylthiazol tetrazolium (MTT) (5 mg/mL in PBS, Sigma-Aldrich, Natick, MA) incorporation on different days. Absorbance at $562 \mathrm{~nm}$ was determined on an ELx800 absorbance microplate reader (Bio-Tek, Winooski, VT). All the growth assays were performed in triplicates and repeated twice.

For three-dimensional cultures, single mouse cancer cells were allowed to form spheroids in 2\% Matrigel (BD Biosciences, San Jose, CA), and the spheroids were transferred to Collagen I extracellular matrix (SigmaAldrich, St. Louis, MO) for 3 days, fixed and stained with antibody for $\beta$-catenin (BD Biosciences, San Jose, CA) and phalloidin (Invitrogen, Carlsbad, CA) for F-actin, countered stained with Sytox Green (Invitrogen, Carlsbad, CA) as described before [29]. Confocal images were captured using a Leica SP5 confocal microscope (Leica Microsystems, Bannockburn, IL) and analyzed by the Leica LAS AF software (Leica Microsystems, Bannockburn, IL).

Total cell lysates were prepared using RIPA lysis buffer (50 mM Tris-HCl, pH 8.0, $150 \mathrm{mM} \mathrm{NaCl}, 1 \%$ Triton X-100, $0.1 \%$ SDS, $0.5 \%$ sodium deoxycholate, supplemented with protease and phosphatase inhibitor cocktail tablets (Roche Diagnostics Corporation, Indianapolis, IN)). Proteins were resolved using standard SDS-PAGE, transferred to PVDF membrane (ThermoFisher Scientific, Waltham, MA) and probed with different antibodies. Pierce ECL Western Blotting substrate (ThermoFisher Scientific, Waltham, MA) was used for signal detection.

\section{RNA extraction, NanoString profiling, and quantitative real-time reverse-transcription PCR (qRT-PCR)}

TRIzol reagent (Life Technologies, Grand Island, NY) was used to extract RNA from cell cultures and tumor tissues. miRNA Expression profiling was performed 
using nCounter miRNA expression assay (Nanostring $®$ Technologies, Seattle, WA). TaqMan MicroRNA Reverse Transcription Kit and TaqMan MicroRNA assay kits (Applied Biosystems, Foster City, CA) were used for qRT-PCR determination of miRNA levels, performed on a 7300 Real-Time PCR System (Applied Biosystems, Foster City, CA). RNU6B RNA was used as the internal control to normalize sample input. Gene expression levels were determined using $2^{-\Delta \Delta \mathrm{CT}}$ method [45].

\section{Introduction of a Dicer1-expression construct into the Dicer-Pten DKO cancer cells and cell growth and adhesion analyses}

A lentiviral FLAG-tagged Dicer1-expression construct EX-H0470-Lv101 was purchased from GeneCopoeia, Inc. (Rockville, MD). Lentiviral transduction particles were prepared by transfecting the lentiviral construct together with ViraPower ${ }^{\mathrm{TM}}$ Lentiviral Packaging Mix (ThermoFisher Scientific, Waltham, MA) into 293FT cells according to the manufacturer's recommendation. The expression of Dicer1 in transduced FTdT172 and FTdT967 tumor cells was confirmed by Western blot analysis using both a FLAG antibody (Cell Signaling Technology, Danvers, MA) and a Dicer1 antibody (Bethyl Laboratories, Montgomery, TX).

For BrdU incorporation and cell cycle analysis, cells were incubated with $10 \mu \mathrm{M}$ of BrdU (Sigma-Aldrich, St. Louis, MO) for $1 \mathrm{~h}$. After trypsinization, cells were fixed with $70 \%$ ethanol and chromosomal DNA was denatured using $2 \mathrm{~N} \mathrm{HCl} / 0.5 \%$ Trion $\mathrm{X}-100$ and neutralized using $0.1 \mathrm{M} \mathrm{Na} \mathrm{B}_{4} \mathrm{O}_{7}$ (Sigma-Aldrich, St. Louis, MO). After resuspension, cells were labeled with FITC-conjugated anti-BrdU antibody (BD Biosciences, San Jose, CA) and $5 \mu \mathrm{g} / \mathrm{mL}$ of propidium iodide (Sigma-Aldrich, St. Louis, $\mathrm{MO}$ ), and analyzed using a BD Accuri ${ }^{\mathrm{TM}} \mathrm{C} 6$ cytometer (BD Biosciences, San Jose, CA).

Cell adhesion assay was performed using the Collagen I cell adhesion strips from Millicoat ${ }^{\mathrm{TM}}$ Screen kit ECM205 (EMD Millipore, Billerica, MA). 1x104 cells were seeded to the strip wells and allowed to incubate for 1 hour. Nonadherent cells were washed away by phosphate buffered saline and the attached cells were stained using $0.2 \%$ crystal violet. The stain was solubilized in a 50:50 mixture of $0.1 \mathrm{M}$ sodium phosphate, $\mathrm{pH} 4.5$ and $50 \%$ ethanol and read at $562 \mathrm{~nm}$. For immunofluorescence, the mouse cells growing in an 8-well chamberslide (BD Biosciences, San Jose, CA) were fixed in 4\% paraformaldehyde (Sigma-Aldrich, St. Louis, MO) and permeabilized with PBS containing $0.5 \%$ Triton X-100 (Sigma-Aldrich, St. Louis, MO). After blocking with 10\% FBS, anti-ZO1 primary antibody (EMD Millipore, Billerica, MA) and Alexa Fluor 647-conjugated antimouse secondary antibody (Invitrogen, Carlsbad, CA) were added sequentially between washes. A mounting medium with DAPI (Vector Laboratories, Burlingame,
CA) was used for counterstaining. Microscopic images were captured by a Leica DM IRE2 fluorescence microscope (Leica Microsystems, Bannockburn, IL) and analyzed by the OpenLab Cell Imaging System software (Leica Microsystems, Bannockburn, IL).

\section{Statistical analysis}

All calculations were performed with MINITAB statistical software (Minitab, State College, PA). Significance of differences was determined using 2-tailed T-Test. A $P$-value of less than 0.05 was considered statistically significant for all tests.

\section{ACKNOWLEDGMENTS}

We acknowledge the support of the Robert and Deborah First Fund, the Sperling Family Fund Foundation, Ruth N. White Gynecologic Oncology Research Fund, Women's Cancer Program and Gillette Center for Women's Cancer from Dana-Farber Cancer Institute, Ovarian Cancer Research Foundation, Adler Foundation, Inc., and Friends of Dana Farber Cancer Institute to The Laboratory of Gynecologic Oncology at Brigham and Women's Hospital. This work is also partly supported by grants awarded to D.M.D. by the DOD OCRP (W81XWH-14-1-0205), American Cancer Society (RSG-13-083-01-TBG), and Ovarian Cancer Research Fund Liz Tilberis award.

\section{CONFLICTS OF INTEREST}

The authors have no potential conflicts of interest to disclose.

\section{REFERENCES}

1. Howlader N, Noone AM, Krapcho M, Garshell J, Miller D, Altekruse SF, Kosary CL, Yu M, Ruhl J, Tatalovich Z, Mariotto A, Lewis DR, Chen HS, Feuer EJ and Cronin KA. (2015). SEER Cancer Statistics Review, 1975-2012. National Cancer Institute, Bethesda, MD).

2. Serov SF and Scullt RE. (1993). Histological typing of ovarian tumors. (Geneva: World Health Organization).

3. Feeley KM and Wells M. Precursor lesions of ovarian epithelial malignancy. Histopathology. 2001; 38:87-95.

4. Auersperg N, Maines-Bandiera SL and Dyck HG. Ovarian carcinogenesis and the biology of ovarian surface epithelium. J Cell Physiol. 1997; 173:261-265.

5. Lee Y, Miron A, Drapkin R, Nucci MR, Medeiros F, Saleemuddin A, Garber J, Birch C, Mou H, Gordon RW, Cramer DW, McKeon FD and Crum CP. A candidate precursor to serous carcinoma that originates in the distal fallopian tube. J Pathol. 2007; 211:26-35. 
6. Crum CP. Intercepting pelvic cancer in the distal fallopian tube: theories and realities. Mol Oncol. 2009; 3:165-170.

7. Kurman RJ and Shih Ie M. The origin and pathogenesis of epithelial ovarian cancer: a proposed unifying theory. Am J Surg Pathol. 2010; 34:433-443.

8. Kuhn E, Kurman RJ, Vang R, Sehdev AS, Han G, Soslow R, Wang TL and Shih Ie M. TP53 mutations in serous tubal intraepithelial carcinoma and concurrent pelvic highgrade serous carcinoma-evidence supporting the clonal relationship of the two lesions. J Pathol. 2012; 226:421-426.

9. Dinulescu DM, Ince TA, Quade BJ, Shafer SA, Crowley D and Jacks T. Role of K-ras and Pten in the development of mouse models of endometriosis and endometrioid ovarian cancer. Nat Med. 2005; 11:63-70.

10. Wu R, Hendrix-Lucas N, Kuick R, Zhai Y, Schwartz DR, Akyol A, Hanash S, Misek DE, Katabuchi H, Williams BO, Fearon ER and Cho KR. Mouse model of human ovarian endometrioid adenocarcinoma based on somatic defects in the Wnt/beta-catenin and PI3K/Pten signaling pathways. Cancer Cell. 2007; 11:321-333.

11. Wu R, Baker SJ, Hu TC, Norman KM, Fearon ER and Cho KR. Type I to type II ovarian carcinoma progression: mutant Trp53 or Pik3ca confers a more aggressive tumor phenotype in a mouse model of ovarian cancer. Am J Pathol. 2013; 182:1391-1399.

12. Flesken-Nikitin A, Choi KC, Eng JP, Shmidt EN and Nikitin AY. Induction of carcinogenesis by concurrent inactivation of p53 and Rb1 in the mouse ovarian surface epithelium. Cancer Res. 2003; 63:3459-3463.

13. Perets R, Wyant GA, Muto KW, Bijron JG, Poole BB, Chin KT, Chen JY, Ohman AW, Stepule CD, Kwak S, Karst AM, Hirsch MS, Setlur SR, Crum CP, Dinulescu DM and Drapkin R. Transformation of the fallopian tube secretory epithelium leads to high-grade serous ovarian cancer in Brca;Tp53;Pten models. Cancer Cell. 2013; 24:751-765.

14. Kim J, Coffey DM, Creighton CJ, Yu Z, Hawkins SM and Matzuk MM. High-grade serous ovarian cancer arises from fallopian tube in a mouse model. Proc Natl Acad Sci U S A. 2012; 109:3921-3926.

15. Zhang L, Ma T, Brozick J, Babalola K, Budiu R, Tseng G and Vlad AM. Effects of Kras activation and Pten deletion alone or in combination on MUC1 biology and epithelial-tomesenchymal transition in ovarian cancer. Oncogene. 2016.

16. Worley MJ, Jr., Liu S, Hua Y, Kwok JS, Samuel A, Hou L, Shoni M, Lu S, Sandberg EM, Keryan A, Wu D, Ng SK, Kuo WP, et al. Molecular changes in endometriosisassociated ovarian clear cell carcinoma. Eur J Cancer. 2015; 51:1831-1842.

17. Mehra K, Mehrad M, Ning G, Drapkin R, McKeon FD, Xian $\mathrm{W}$ and Crum CP. STICS, SCOUTs and p53 signatures; a new language for pelvic serous carcinogenesis. Front Biosci (Elite Ed). 2011; 3:625-634.

18. Hill DA, Ivanovich J, Priest JR, Gurnett CA, Dehner LP, Desruisseau D, Jarzembowski JA, Wikenheiser-Brokamp
KA, Suarez BK, Whelan AJ, Williams G, Bracamontes D, Messinger Y and Goodfellow PJ. DICER1 mutations in familial pleuropulmonary blastoma. Science. 2009; 325:965.

19. Doros L, Yang J, Dehner L, Rossi CT, Skiver K, Jarzembowski JA, Messinger Y, Schultz KA, Williams G, Andre $\mathrm{N}$ and Hill DA. DICER1 mutations in embryonal rhabdomyosarcomas from children with and without familial PPB-tumor predisposition syndrome. Pediatr Blood Cancer. 2012; 59:558-560.

20. Rio Frio T, Bahubeshi A, Kanellopoulou C, Hamel N, Niedziela M, Sabbaghian N, Pouchet C, Gilbert L, O'Brien PK, Serfas K, Broderick P, Houlston RS, Lesueur F, et al. DICER1 mutations in familial multinodular goiter with and without ovarian Sertoli-Leydig cell tumors. JAMA. 2011; 305:68-77.

21. Heravi-Moussavi A, Anglesio MS, Cheng SW, Senz J, Yang W, Prentice L, Fejes AP, Chow C, Tone A, Kalloger SE, Hamel N, Roth A, Ha G, et al. Recurrent somatic DICER1 mutations in nonepithelial ovarian cancers. N Engl J Med. 2012; 366:234-242.

22. Chen J, Wang Y, McMonechy MK, Anglesio MS, Yang W, Senz J, Maines-Bandiera S, Rosner J, Trigo-Gonzalez G, Grace Cheng SW, Kim J, Matzuk MM, Morin GB and Huntsman DG. Recurrent DICER1 hotspot mutations in endometrial tumours and their impact on microRNA biogenesis. J Pathol. 2015; 237:215-225.

23. Drapkin R, Crum CP and Hecht JL. Expression of candidate tumor markers in ovarian carcinoma and benign ovary: evidence for a link between epithelial phenotype and neoplasia. Hum Pathol. 2004; 35:1014-1021.

24. Pietzner K, Woopen H, Richter R, Joens T, Braicu EI, Dimitrova D, Mellstedt H, Darb-Esfahani S, Denkert C, Lindhofer H, Fotopoulou C and Sehouli J. Expression of epithelial cell adhesion molecule in paired tumor samples of patients with primary and recurrent serous ovarian cancer. Int J Gynecol Cancer. 2013; 23:797-802.

25. Rodriguez M and Dubeau L. Ovarian tumor development: insights from ovarian embryogenesis. Eur J Gynaecol Oncol. 2001; 22:175-183.

26. Sundfeldt K, Piontkewitz Y, Ivarsson K, Nilsson O, Hellberg P, Brannstrom M, Janson PO, Enerback S and Hedin L. E-cadherin expression in human epithelial ovarian cancer and normal ovary. Int J Cancer. 1997; 74:275-280.

27. Auersperg N, Pan J, Grove BD, Peterson T, Fisher J, Maines-Bandiera S, Somasiri A and Roskelley CD. E-cadherin induces mesenchymal-to-epithelial transition in human ovarian surface epithelium. Proc Natl Acad Sci U S A. 1999; 96:6249-6254.

28. Ong A, Maines-Bandiera SL, Roskelley CD and Auersperg N. An ovarian adenocarcinoma line derived from SV40/ E-cadherin-transfected normal human ovarian surface epithelium. Int J Cancer. 2000; 85:430-437. 
29. Choi PW, Yang J, Ng SK, Feltmate C, Muto MG, Hasselblatt K, Lafferty-Whyte K, JeBailey L, MacConaill L, Welch WR, Fong WP, Berkowitz RS and Ng SW. Loss of E-cadherin disrupts ovarian epithelial inclusion cyst formation and collective cell movement in ovarian cancer cells. Oncotarget. 2016; 7:4110-4121. doi: 10.18632/ oncotarget.6588.

30. Karst AM, Levanon K and Drapkin R. Modeling high-grade serous ovarian carcinogenesis from the fallopian tube. Proc Natl Acad Sci U S A. 2011; 108:7547-7552.

31. Mihaylova MM and Shaw RJ. The AMPK signalling pathway coordinates cell growth, autophagy and metabolism. Nat Cell Biol. 2011; 13:1016-1023.

32. Ryeom SW, Paul D and Goodenough DA. Truncation mutants of the tight junction protein ZO-1 disrupt corneal epithelial cell morphology. Mol Biol Cell. 2000; 11:1687-1696.

33. Frezzetti D, Reale C, Cali G, Nitsch L, Fagman H, Nilsson O, Scarfo M, De Vita G and Di Lauro R. The microRNAprocessing enzyme Dicer is essential for thyroid function. PLoS One. 2011; 6:e27648.

34. Singh MK, Lu MM, Massera D and Epstein JA. MicroRNAprocessing enzyme Dicer is required in epicardium for coronary vasculature development. J Biol Chem. 2011; 286:41036-41045.

35. Woiwode A, Johnson SA, Zhong S, Zhang C, Roeder RG, Teichmann $\mathrm{M}$ and Johnson DL. PTEN represses RNA polymerase III-dependent transcription by targeting the TFIIIB complex. Mol Cell Biol. 2008; 28:4204-4214.

36. Palian BM, Rohira AD, Johnson SA, He L, Zheng N, Dubeau L, Stiles BL and Johnson DL. Mafl is a novel target of PTEN and PI3K signaling that negatively regulates oncogenesis and lipid metabolism. PLoS Genet. 2014; 10:e1004789.
37. Johnson SA, Dubeau L and Johnson DL. Enhanced RNA polymerase III-dependent transcription is required for oncogenic transformation. J Biol Chem. 2008; 283:19184-19191.

38. Johnson DL and Johnson SA. Cell biology. RNA metabolism and oncogenesis. Science. 2008; 320:461-462.

39. Tang Y, Lin Y, Li C, Hu X, Liu Y, He M, Luo J, Sun G, Wang T, Li W and Guo M. MicroRNA-720 promotes in vitro cell migration by targeting Rab35 expression in cervical cancer cells. Cell Biosci. 2015; 5:56.

40. Guzman N, Agarwal K, Asthagiri D, Yu L, Saji M, Ringel MD and Paulaitis ME. Breast Cancer-Specific miR Signature Unique to Extracellular Vesicles Includes "microRNA-like" tRNA Fragments. Mol Cancer Res. 2015; 13:891-901.

41. Kong J, Liu X, Li X, Wu J, Wu N, Chen J and Fang F. miR-125/Pokemon auto-circuit contributes to the progression of hepatocellular carcinoma. Tumour Biol. 2016; 37:511-519.

42. Cancer Genome Atlas Research N. Integrated genomic analyses of ovarian carcinoma. Nature. 2011; 474:609-615.

43. Chen CZ, Li L, Lodish HF and Bartel DP. MicroRNAs modulate hematopoietic lineage differentiation. Science. 2004; 303:83-86.

44. Huang KC, Park DC, Ng SK, Lee JY, Ni X, Ng WC, Bandera CA, Welch WR, Berkowitz RS, Mok SC and Ng $\mathrm{SW}$. Selenium binding protein 1 in ovarian cancer. Int $\mathrm{J}$ Cancer. 2006; 118:2433-2440.

45. Huang KC, Rao PH, Lau CC, Heard E, Ng SK, Brown C, Mok SC, Berkowitz RS and Ng SW. Relationship of XIST expression and responses of ovarian cancer to chemotherapy. Mol Cancer Ther. 2002; 1:769-776. 